\title{
Simple current symmetries in RCFT
}

\author{
P. Bantay \\ Institute for Theoretical Physics, Eotvos University Budapest
}

26th November 2018

\begin{abstract}
The question "Which abelian permutation groups arise as group of simple currents in Rational Conformal Field Theory?" is investigated using the formalism of weighted permutation actions. After a review of the relevant properties of simple current symmetries, the general theory of WPA-s and admissibility conditions are described, and classification results are illustrated by a couple of examples.
\end{abstract}

\section{Introduction}

The global structure of a Rational Conformal Field Theory is described by a Modular Tensor Category [1, 2, i.e. a solution of the Moore-Seiberg polynomial equations [3] and the associated modular representation. Besides the behavior of the genus one characters under the action of the modular group [4, the Modular Tensor Category does also determine the fusion rules of the primary fields [5], the modular invariant partition functions [6], the conformal boundary conditions [7] [8, and many other important characteristics of the theory.

Recall that the modular representation associated to a RCFT is determined by a pair of unitary matrices $S$ and $T$ representing the modular transformations $\tau \mapsto \frac{-1}{\tau}$ and $\tau \mapsto \tau+1$ respectively (with rows and columns indexed by the primary fields), such that [3, 5]

1. $T$ is diagonal of finite order, and $S$ is symmetric;

2. $S^{2}$ commutes with $T$, and equals the permutation matrix associated to charge conjugation;

3. the modular relation

$$
S T S=T^{-1} S T^{-1}
$$

holds.

The eigenvalues of the matrix $T$ are determined by the conformal weights $\Delta_{p}$ of the primaries and the central charge $c$, more precisely

$$
T_{p}^{q}=\omega(p) \delta_{p}^{q}
$$


with

$$
\omega(p)=\exp \left(2 \pi \imath\left(\Delta_{p}-\frac{c}{24}\right)\right),
$$

while the matrix elements of $S$ determine the fusion rules $N_{p q r}$ of the primaries by Verlinde's formula [ 5

$$
N_{p q r}=\sum_{s} \frac{S_{p s} S_{q s} S_{r s}}{S_{0 s}}
$$

where 0 labels the vacuum.

Symmetries of the modular representation have been studied extensively, mostly because of their intimate ties with the classification of modular invariant partition functions. A prominent role is played by simple current symmetries, which are permutations $\alpha$ of the primary fields for which the associated permutation matrix

$$
[\Pi(\alpha)]_{p}^{q}=\delta_{p}^{\alpha q}
$$

is diagonalized by $S$. The terminology comes from the relation of such permutations to simple currents, which are defined traditionally as primary fields $J$ whose fusion product with their charge conjugate equals the vacuum [9, 10, 11, 12, a condition that may be shown to be equivalent to $S_{0 J}=S_{00}$. From this definition follows, using standard properties of the modular representation and Verlinde's formula Eq. (4), that the fusion product of two simple currents is again a simple current, i.e. they form an abelian group, and that the fusion matrix $N_{J}$ associated to a simple current $J$ is a permutation matrix. In other words, to any simple current $J$ is associated a permutation $\alpha_{J}$ of the primary fields such that $N_{J}=\Pi\left(\alpha_{J}\right)$. According to Verlinde's theorem [5] fusion matrices are diagonalized by $S$, consequently $\alpha_{J}$ is a simple current symmetry for each $J$. Conversely, $\alpha 0$ is a simple current for each simple current symmetry $\alpha$, and this one-to-one correspondence between simple currents and simple current symmetries shows that the two notions are in fact equivalent. We'll use the definition based on permutations because it is more adequate for the kind of questions we intend to discuss.

Simple currents play a distinguished role in Conformal Field Theory for several reasons. Just to cite a few, they provide for most modular invariant partition functions [13, 14, they are essential for the proper treatment of the GSO projection in Superstring Theory [15, 16, 17, they can be used to construct new non-trivial modular tensor categories [18, and they determine to a great extent the structure of the modular representation [19. All these applications need a thorough and detailed understanding of their properties.

While there is an extensive literature on simple currents 9, 10, 11, 12, 20, 21, 22], the following question still waits for an answer.

Question1: Which finite abelian permutation groups are group of simple current symmetries of a RCFT?

That any (finite) abelian group arises this way is easy to see, but it is far from true that any abelian permutation group does. For example, we'll see that the 
degree of the group of simple current symmetries is bounded from below by its order, restricting severely the possible permutation groups.

A simple minded approach to answering Question1 would be to classify all RCFT-s (or the corresponding modular tensor categories), and to compute the group of simple current symmetries for each of them: sorting the resulting list would yield the answer. Unfortunately, the enumeration of all RCFT-s is still beyond reach, so we need some alternative strategy. The one we'll adopt here is to look for properties that are characteristic of the permutation action of the group of simple current symmetries, then, for a prescribed abelian group, to classify all permutation actions with these properties. As we'll see, these characteristic properties are related to the extra structure of simple current symmetries, formalized in the notion of an admissible weighted permutation action. In this way we'll get an answer to our problem, which is only partial because not all admissible WPA-s correspond to simple current symmetries of RCFT-s. Nevertheless, this partial answer will already allow us to draw surprising conclusions about RCFT-s with a prescribed group of simple current symmetries, e.g. that a theory which has a $\mathbb{Z}_{3} \times \mathbb{Z}_{3}$ group of integer spin simple currents should have at least 35 primary fields (Example 4 of Section 5 ), a result that is not completely obvious. But first of all, we have to take a closer look at simple current symmetries.

\section{Simple current symmetries}

As explained in Section 1 a simple current symmetry is a permutation $\alpha$ of the primary fields such that $S^{-1} \Pi(\alpha) S$ is a diagonal matrix, where $\Pi(\alpha)$ denotes the permutation matrix associated to $\alpha$. A straightforward argument shows that simple current symmetries form an abelian group $G$ (commutativity being a consequence of the commutativity of diagonal matrices). The importance of simple current symmetries is related to the following result, somewhat reminiscent of Schur's lemma: any monomial matrix (i.e. a matrix that has exactly one nonzero entry in each row and column) diagonalized by $S$ is necessarily proportional to $\Pi(\alpha)$ for some $\alpha \in G$.

The basic properties of simple current symmetries are neatly expressed in terms of the monomial matrices

$$
Y(\alpha, \beta)=\Pi(\alpha) S \Pi(\beta) S^{-1} .
$$

A clever application of the modular relation Eq.(1), together with Eq.(2) and the symmetry of $S$, leads to the formula

$$
Y(\alpha, \beta)_{p}^{q}=\vartheta(\beta) \frac{\omega(q)}{\omega(\beta q)} \delta_{p}^{\alpha q}
$$

for the matrix elements, where

$$
\vartheta(\alpha)=\exp \left(2 \pi \imath \Delta_{\alpha 0}\right)
$$


Inserting Eq. (17) into the obvious equality $Y(1, \alpha) Y(1, \beta)=Y(1, \alpha \beta)$ gives the fundamental relation

$$
\frac{\omega(\alpha p) \omega(\beta p)}{\omega(p) \omega(\alpha \beta p)}=\frac{\vartheta(\alpha) \vartheta(\beta)}{\vartheta(\alpha \beta)}
$$

which holds for all primaries $p$ and all $\alpha, \beta \in G$, where the point is that the lhs. of Eq. (9) does not depend on $p$.

Eq.(9) is the cornerstone of the whole theory. In particular, from Eqs.(7) and (9) one derives the multiplication rule

$$
Y\left(\alpha_{1}, \beta_{1}\right) Y\left(\alpha_{2}, \beta_{2}\right)=\frac{\vartheta\left(\alpha_{2}\right) \vartheta\left(\beta_{1}\right)}{\vartheta\left(\alpha_{2} \beta_{1}\right)} Y\left(\alpha_{1} \alpha_{2}, \beta_{1} \beta_{2}\right) .
$$

In other words the matrices $Y(\alpha, \beta)$ form a projective representation of the abelian group $G \times G$, with a suitable 2-cocycle determined by $\vartheta$. In terms of $\vartheta$ the 2-cocycle condition reads

$$
\vartheta(\alpha \beta) \vartheta(\beta \gamma) \vartheta(\gamma \alpha)=\vartheta(\alpha) \vartheta(\beta) \vartheta(\gamma) \vartheta(\alpha \beta \gamma),
$$

i.e. $\vartheta$ is a quadratic function on $G$ written multiplicatively! The 2-cocycle of Eq. (10) is trivial if and only if $\vartheta$ is a character of $G$, i.e. $\vartheta(\alpha \beta)=\vartheta(\alpha) \vartheta(\beta)$. One may also show that in a unitary RCFT

$$
\vartheta\left(\alpha^{n}\right)=\vartheta(\alpha)^{n^{2}}
$$

holds for all integers $n$ and $\alpha \in G$, i.e. $\vartheta$ is actually a quadratic form on $G$, a well known result of the theory of simple currents [9, 11].

Let's now consider the commutation rule of $Y(\alpha, \beta)$ with modular representation matrices. If $M$ represents the $S L(2, \mathbb{Z})$ element $\left(\begin{array}{ll}a & b \\ c & d\end{array}\right)$, then

$$
M^{-1} Y(\alpha, \beta) M=\frac{\vartheta(\alpha)^{b(c-a)} \vartheta(\beta)^{c(b-d)}}{\vartheta(\alpha \beta)^{b c}} Y\left(\alpha^{a} \beta^{c}, \alpha^{b} \beta^{d}\right) .
$$

In particular,

$$
S^{-1} Y(\alpha, \beta) S=\frac{\vartheta(\alpha \beta)}{\vartheta(\alpha) \vartheta(\beta)} Y\left(\beta, \alpha^{-1}\right)
$$

and

$$
T^{-1} Y(\alpha, \beta) T=\frac{1}{\vartheta(\alpha)} Y(\alpha, \alpha \beta) .
$$

Eq.(13) is the basis of the application of simple currents to the construction of modular invariant partition functions [13, 14]: if $H<G$ is a subgroup such that $\vartheta(\alpha)=1$ for all $\alpha \in H$ (i.e. $H$ corresponds to integer spin simple currents), then the matrix

$$
Z_{H}=\frac{1}{|H|} \sum_{\alpha, \beta \in H} Y(\alpha, \beta)
$$


commutes with all modular matrices according to Eq.(7), and may be shown to have non-negative integer entries, thus providing a modular invariant, the socalled simple current modular invariant associated to the subgroup $H$. There is a further possibility to introduce into Eq.(16) so-called "discrete torsion" coefficients [14.

An important feature of the matrices $Y(\alpha, \beta)$, which goes beyond the usual properties of the modular representation, follows from considerations involving the mapping class group action on the space of genus one 1-point holomorphic blocks, more precisely from the "curious relation" Eq.(33) of [23]. According to this result, the trace of $Y(\alpha, \beta)$ may be expressed as

$$
\operatorname{Tr} Y(\alpha, \beta)=\sum_{p} \phi_{p}(\alpha, \beta)
$$

where the quantity $\phi_{p}(\alpha, \beta)$, the so-called "commutator cocycle", satisfies

$$
\left|\phi_{p}(\alpha, \beta)\right|= \begin{cases}1 & \text { if } p \text { is fixed by both } \alpha \text { and } \beta, \\ 0 & \text { otherwise. }\end{cases}
$$

Finally, one may show that no simple current symmetry besides the identity may fix the vacuum. The importance of this remark will be clarified in Section 4. but it does already imply that there are at least as many primary fields as simple currents, i.e. the degree of the permutation group $G$ cannot be less than its order.

\section{$3 \quad$ Weighted permutation actions}

By a quadratic group we'll mean a pair $(G, \vartheta)$, with $\vartheta$ a quadratic function on the finite abelian group $G$, i.e. a complex valued function $\vartheta: G \rightarrow \mathbb{C}^{*}$ such that Eq.(11) is satisfied. Its radical is the quadratic group

$$
\operatorname{rad}(G, \vartheta)=(\sqrt{G}, \sqrt{\vartheta})
$$

where $\sqrt{G}=\{\alpha \in G \mid \vartheta(\alpha \beta)=\vartheta(\alpha) \vartheta(\beta) \quad \forall \beta \in G\}$ and $\sqrt{\vartheta}$ is the restriction of $\vartheta$ to $\sqrt{G}$. Note that $\sqrt{\vartheta}$, besides being a quadratic function, is also a character of $\sqrt{G}$, i.e. it is a homomorphism from $\sqrt{G}$ into the complex numbers. A quadratic group is called non-degenerate if its radical is trivial, while it is called completely degenerate if it equals its own radical (in which case the quadratic function $\vartheta$ is a character of $G$ ). While usually the nondegenerate case is the interesting one, we'll see that in the present context it's just the opposite: all the interest lies in the completely degenerate case.

By a weighted permutation action (WPA for short) of a quadratic group $(G, \vartheta)$ we shall mean a pair $\mathcal{X}=(X, \omega)$, where $X$ (the support of $\mathcal{X})$ is a finite set permuted by the group $G$, while $\omega$ (the weight function of $\mathcal{X}$ ) is a nowhere vanishing complex function on $X$ which satisfies Eq. (9). The degree of the WPA 
$(X, \omega)$ is the cardinality of $X$. According to the results of Section 2 the group $G$ of simple current symmetries of a RCFT, together with the quadratic function $\vartheta$ defined by Eq. (8), form a quadratic group $(G, \vartheta)$, and the pair $(X, \omega)$ is a weighted permutation action of this quadratic group, where $X$ denotes the set of primary fields and $\omega$ is defined by Eq.(3). We shall call this WPA the simple current WPA associated to the RCFT.

Clearly, for a given WPA $\mathcal{X}=(X, \omega)$ of the quadratic group $(G, \vartheta)$ the labeling of the elements of $X$ is immaterial, leading to an obvious notion of equivalence for WPA-s, with an additional freedom: Eq.(9) is invariant under a rescaling of $\omega$, therefore two WPA-s whose weight functions differ by a factor which is locally constant on the orbits of $G$ should be considered equivalent too. Our goal in this Section is to understand the classification of WPA-s up to equivalence.

First of all, let's see an important example of a WPA. For a given quadratic group $(G, \vartheta)$ let's consider the WPA whose support is the set of elements of $G$ (on which $G$ acts by left translations), and whose weight function equals $\vartheta$. That this is indeed a WPA, which we'll call the regular WPA, follows from Eq. (11). This WPA is transitive, which means that its support consist of just one $G$-orbit, and it will play an especially important role later.

Given two WPA-s $\mathcal{X}_{1}=\left(X_{1}, \omega_{1}\right)$ and $\mathcal{X}_{2}=\left(X_{2}, \omega_{2}\right)$ with disjoint supports (i.e. such that $X_{1} \cap X_{2}=\emptyset$ ), their sum is the WPA denoted $\mathcal{X}_{1} \oplus \mathcal{X}_{2}$, with support $X_{1} \cup X_{2}$ and weight function

$$
\omega_{\mathcal{X}}(p)= \begin{cases}\omega_{1}(p) & \text { if } p \in X_{1} \\ \omega_{2}(p) & \text { if } p \in X_{2}\end{cases}
$$

It may be shown that one gets in this way a commutative and associative binary operation on the set of equivalence classes of WPA-s. There is also an obvious notion of product of WPA-s, but it won't play any role in what follows.

Transitive WPA-s, i.e. those WPA-s whose support consist of a single $G$ orbit, are fundamental because any WPA may be decomposed uniquely (up to reordering) into a sum of transitive ones. This result reduces the classification of (equivalence classes of) WPA-s to the problem of determining the transitives.

All transitive WPA-s may be obtained (up to equivalence) as follows: let $\xi$ be a character of $\sqrt{G}$, and $H$ a subgroup of $\operatorname{ker}(\xi / \sqrt{\vartheta})$ (the latter makes sense since $\sqrt{\vartheta}$ is a character of $\sqrt{G})$. Consider the coset space $X=G / H$ on which $G$ acts by left translations, and the function $\omega(\alpha H)=\vartheta(\alpha) / \xi^{*}(\alpha)$, where $\xi^{*}$ is any character of $G$ whose restriction to $\sqrt{G}$ equals $\xi$. One may show that this $\omega$ is well-defined on $G / H$, and the pair $(X, \omega)$ is a transitive WPA, whose equivalence class $\mathcal{W}[H, \xi]$ is fully determined by $H$ and $\xi$, and any transitive WPA is equivalent to one of these coset WPA-s. The regular WPA is just $\mathcal{W}\left[1, \xi_{0}\right]$, where $\xi_{0}$ denotes the trivial character, and 1 is the trivial subgroup of $G$.

There is a result that simplifies greatly the classification of WPA-s. It states that there is a one-to-one correspondence between the WPA-s of a quadratic 
group and those of its radical, which respects sums, and under which transitive WPA-s correspond to transitive ones! In particular, a non-degenerate quadratic group has just one transitive WPA (the regular one), all other WPA-s being multiples of it, showing that, as far as WPA-s are concerned, the interest does indeed lie in the completely degenerate case.

In the same way as one can associate a linear representation to any permutation action of a group, one can associate a monomial representation to a WPA $(X, \omega)$ by introducing matrices

$$
Y(\alpha, \beta)_{p}^{q}=\vartheta(\beta) \frac{\omega(q)}{\omega(\beta q)} \delta_{p}^{\alpha q},
$$

whose rows and columns are labeled by the elements of $X$. These matrices form a projective representation of $G \times G$, i.e.

$$
Y\left(\alpha_{1}, \beta_{1}\right) Y\left(\alpha_{2}, \beta_{2}\right)=\frac{\vartheta\left(\alpha_{2}\right) \vartheta\left(\beta_{1}\right)}{\vartheta\left(\alpha_{2} \beta_{1}\right)} Y\left(\alpha_{1} \alpha_{2}, \beta_{1} \beta_{2}\right) .
$$

Moreover, denoting by $A^{t}$ the transpose of a matrix $A$, it follows from Eqs.(21) and (9) that

$$
Y(\alpha, \beta)^{t}=\frac{\vartheta(\alpha \beta)}{\vartheta(\alpha) \vartheta(\beta)} Y\left(\alpha^{-1}, \beta\right)
$$

The quantity

$$
\Upsilon_{\mathcal{X}}(\alpha, \beta)=\vartheta(\beta) \sum_{p \in \operatorname{Fix}(\alpha)} \frac{\omega(p)}{\omega(\beta p)}=\operatorname{Tr} Y(\alpha, \beta)
$$

where Fix $(\alpha)$ denotes the set of fixed points of the permutation $\alpha$, will play an important role in Section 4 It is obviously additive, i.e.

$$
\Upsilon_{\mathcal{X}}(\alpha, \beta)=\Upsilon_{\mathcal{X}_{1}}(\alpha, \beta)+\Upsilon_{\mathcal{X}_{2}}(\alpha, \beta)
$$

if $\mathcal{X}=\mathcal{X}_{1} \oplus \mathcal{X}_{2}$, and for the transitive WPA $\mathcal{W}[H, \xi]$ (see Section 3 ) one has

$$
\Upsilon(\alpha, \beta)= \begin{cases}\xi(\beta)[G: H] & \text { if } \alpha \in H \text { and } \beta \in \sqrt{G}, \\ 0 & \text { otherwise. }\end{cases}
$$

Note that $\Upsilon(\alpha, \beta)=0$ unless $\alpha, \beta \in \sqrt{G}$, consequently we may consider $\Upsilon$ as a function defined on $\sqrt{G} \times \sqrt{G}$.

\section{Admissible WPAs}

As we have already discussed in Section 3, to each RCFT corresponds a WPA $(X, \omega)$ of the quadratic group $(G, \vartheta)$, where $X$ is the set of primary fields, $\omega$ is defined by Eq.(3), $G$ is the group of simple current symmetries, and $\vartheta$ the quadratic function defined by Eq.(8). In view of this result, we may reformulate Question1 from Section 1 as follows. 
Question2: For a given a quadratic group, which of its WPA-s arise as simple current WPA-s of some RCFT?

It is clear that an arbitrary WPA won't do the job. First, because the vacuum 0 has trivial stabilizer (as noticed at the end of Section 21), and the weight function $\omega$ restricted to the $G$-orbit of 0 equals, up to an irrelevant constant of proportionality, the quadratic form $\vartheta$ according to Eqs.(3) and (8), it follows that the simple current WPA of a unitary RCFT should contain at least one copy of the regular WPA. But this is not the end of the story.

An analysis of the properties of simple current WPA-s leads to the notion of admissible WPA, by which we mean a WPA $\mathcal{X}$ that satisfies the following criteria:

1. Galois invariance:

$$
\operatorname{mult}_{\mathcal{X}}\left(H, \xi^{k}\right)=\operatorname{mult}_{\mathcal{X}}(H, \xi)
$$

for any integer $k$ coprime to the exponent of $G$, where mult $\mathcal{X}(H, \xi)$ denotes the multiplicity of the transitive WPA $\mathcal{W}[H, \xi]$ in $\mathcal{X}$.

2. Reciprocity relation:

$$
\Upsilon(\beta, \alpha)=\Upsilon(\alpha, \beta) .
$$

3. Fixed point bound:

$$
|\Upsilon(\alpha, \beta)| \leq|\operatorname{Fix}(\alpha) \cap \operatorname{Fix}(\beta)|
$$

for all $\alpha, \beta \in G$, i.e. the absolute value of $\Upsilon(\alpha, \beta)$ is bounded from above by the number of common fixed points of $\alpha$ and $\beta$.

Let's explain the origin of these conditions! Eq.(27) follows ultimately from Eq. (13), for one can show using the theory of the Galois action [24, 25], that the matrix $S^{-1} T^{l} S T^{k} S T^{l}$ is monomial for integers $l$ and $k$ such that $l k \equiv$ $1(\bmod N)$, where $N$ denotes the order of the matrix $T[26]$. Because the exponent of the group $G$ of simple currents divides $N$, Eq.(13) applied to such matrices $S^{-1} T^{l} S T^{k} S T^{l}$, taking into account their monomiality, leads to Eq.(27) for the simple current WPA of a RCFT.

As to Eq.(28), it comes from combining Eqs.(14) and (23), which give

$$
S^{-1} Y(\alpha, \beta)^{t} S=Y(\beta, \alpha) .
$$

Taking the trace of both sides and recalling Eq.(24) gives at once the reciprocity relation Eq.(28). Finally, the fixed point bound follows from Eqs.(17), (18) and the triangle inequality.

According to the above terminology, the simple current WPA of a unitary RCFT is an admissible WPA which contains the regular WPA with positive multiplicity. Note that the above admissibility conditions are necessary, but by no means sufficient! 
One might wonder how restrictive is the above concept of admissibility, and on the possible dependencies between Eqs.(27), (28) and (29). One may show that they are actually independent by exhibiting relatively simple examples which satisfy two of them, but not the third. As to the strength of these restrictions, let's just mention the following result: for any WPA that satisfies the reciprocity relation Eq.(28), the values of $\Upsilon(\alpha, \beta)$ are always integers! The examples from Section 5 further illustrate the special nature of admissible WPA-s. In principle one could develop more restrictive criteria for WPA-s corresponding to simple current symmetries of a RCFT, but the above notion of admissibility has a distinctive feature: it puts linear restrictions (equalities and inequalities) on the multiplicities of the transitive constituents, which means that the sum of two admissible WPA-s is again admissible.

Let's turn now to the classification of admissible WPA-s. For this we'll need the notion of irreducibility: a WPA is irreducible if it is admissible and cannot be decomposed into a non-trivial sum of two admissible WPA-s. One can show that there is only a finite number of irreducibles, which follows from the fact that the admissibility conditions translate into a set of homogeneous linear equalities and inequalities on the multiplicities of the transitive constituents, and these define a rational cone which, according to the celebrated theorem of Minkowski and Weyl [27], is always generated by a finite number of extreme rays .

Consequently, it is in principle possible to determine for each quadratic group the set of irreducible WPA-s, and any admissible WPA will arise as a sum of these irreducibles. But it should be noticed that, although an irreducible decomposition always exists for an admissible WPA, this decomposition is by no means unique, for there may exist non-trivial relations among the irreducibles (as in Example 4 of the next Section).

An important simplification results from the one-to-one correspondence (discussed in Section (3) between the WPA-s of a quadratic group and those of its radical, for irreducible WPA-s correspond under it to irreducible ones. Thus it is enough to classify the irreducibles WPA-s of the radical. In particular, for a nondegenerate quadratic group the regular WPA, which is the only transitive, is also the only irreducible, which corresponds to the situation where all primaries are simple currents. Conversely, the regular WPA cannot be admissible unless the quadratic group is nondegenerate.

\section{$5 \quad$ Examples}

As indicated in the main text, there is a one-to-one correspondence between the WPA-s of a quadratic group and the WPA-s of its radical, under which transitive (resp. irreducible) WPA-s correspond to transitive (resp. irreducible) ones. Thus it is enough to treat the completely degenerate case, when the quadratic function is a character. As usual, $\mathbb{Z}_{n}$ is the cyclic group of order $n$, and we'll denote by $\xi_{0}$ the trivial character of any group, while $\xi_{1}$ stands for the non-trivial character of $\mathbb{Z}_{2}$. 
1. $\mathcal{G}=\left(\mathbb{Z}_{2}, \xi_{0}\right)$.

This quadratic group has three transitive WPA-s (see Section 3 for notations): $F=\mathcal{W}\left[\mathbb{Z}_{2}, \xi_{0}\right]$ of degree 1 (the fixed point), $R=\mathcal{W}\left[1, \xi_{0}\right]$ of degree 2 (the regular), and $W=\mathcal{W}\left[1, \xi_{1}\right]$ of degree 2 . The Galois action is trivial because $G$ has exponent 2. A WPA

$$
n_{F} F \oplus n_{R} R \oplus n_{W} W,
$$

is admissible if and only if the following conditions are satisfied:

$$
\begin{aligned}
n_{R} & =n_{W}, \\
n_{F}, n_{R} & \geq 0 .
\end{aligned}
$$

It does follow that there are two irreducible WPA-s: $F$ of degree 1 and $R \oplus$ $W$ of degree 4 , and there are no relations between them. Any admissible WPA that contains the regular one does also contain the WPA $W$, in particular its degree is at least 4 .

2. $\mathcal{G}=\left(\mathbb{Z}_{2}, \xi_{1}\right)$.

Again we have three transitive WPA-s, $F=\mathcal{W}\left[\mathbb{Z}_{2}, \xi_{1}\right], R=\mathcal{W}\left[1, \xi_{0}\right]$ and $W=\mathcal{W}\left[1, \xi_{1}\right]$, out of which $R$ is the regular of degree 2 , and $F$ is the fixed-point WPA of degree 1 . The admissibility conditions for $n_{F} F \oplus$ $n_{R} R \oplus n_{W} W$ read

$$
\begin{aligned}
n_{R} & =n_{F}+n_{W}, \\
n_{F}, n_{W} & \geq 0 .
\end{aligned}
$$

Consequently, there are two irreducible WPA-s: $F \oplus R$ of degree 3 and $R \oplus W$ of degree 4 , and there is no relation between them. In this case both irreducibles contain the regular WPA $R$. The irreducible $F \oplus R$ is realized in the Ising model.

3. $\mathcal{G}=\left(\mathbb{Z}_{2} \times \mathbb{Z}_{2}, \xi_{0}\right)$.

In this case there are 11 transitive WPA-s. Solving the conditions for admissibility, we get a total of 6 irreducible WPA-s, out of which three have degree 4 , and the remaining three have degrees 1,11 and 16 respectively. There are no relations between the irreducibles. The only irreducibles that contain the regular WPA are those of degrees 11 and 16. The irreducible of degree 11 is realized in a suitable Ashkin-Teller model.

4. $\mathcal{G}=\left(\mathbb{Z}_{3} \times \mathbb{Z}_{3}, \xi_{0}\right)$.

There are 22 transitive WPA-s belonging to 14 Galois orbits, but only 8 irreducible ones. Of the irreducible WPA-s four have degree 9 , while the remaining have respective degrees $1,35,69$ and 81 . The only irreducible WPA-s containing the regular one are those of degrees 35, 69 and 81 . There is one non-trivial relation between the irreducibles, which reads $\underline{35} \oplus \underline{35}=\underline{1} \oplus \underline{69}$ if $\underline{d}$ denotes the unique irreducible of degree $d$. 
While the simplest cases with $G=\mathbb{Z}_{2}$ may be dealt with by hand, the larger cases (large means $|G|>3$ ) require the use of sophisticated software. The needed group theoretic computations have been performed using GAP [28, while the solution of the inequalities coming from Eq.(29) were computed by the software package cdd +29 . It should be noted that this last part of the computations is in general extremely hard. For example, in the cases $\mathcal{G}=\left(\mathbb{Z}_{2} \times \mathbb{Z}_{2} \times \mathbb{Z}_{2}, \xi_{0}\right)$ and $\mathcal{G}=\left(\mathbb{Z}_{4} \times \mathbb{Z}_{4}, \xi_{0}\right)$ more than 800 hours of CPU time have not been enough to complete the computation on a $2.4 \mathrm{GHz}$ Pentium4 PC.

\section{Discussion}

The main goal of this work was to isolate the notions needed for a proper treatment of the question: which finite abelian permutation groups arise as group of simple current symmetries? The analysis of the properties of simple current symmetries led us to introduce the notions of weighted permutation actions and admissibility, and to develop the ensuing theory. While admissibility is a necessary but not sufficient condition for a WPA to be the simple current WPA of some RCFT, it has the enormous advantage of being linear in the multiplicities of the transitive constituents, which greatly simplifies the theory. Unfortunately, the solution of the linear inequalities coming from the fixed point bound Eq.(29) turns out to be very involved even in simple cases like $G=\mathbb{Z}_{2} \times$ $\mathbb{Z}_{2} \times \mathbb{Z}_{2}$. But in principle we can determine in finite time the irreducible WPA-s of any quadratic group, together with the relations satisfied by them, providing a complete solution of the admissibility conditions and an answer to Question2. While the notion admissibility is not restrictive enough, i.e. it may happen

that some admissible WPA-s do not arise from a RCFT, it does nevertheless capture many non-trivial features of simple current WPA-s, hopefully the most important ones.

Work supported by grants OTKA T32453, T47041, T37674 and T43582.

\section{References}

[1] V.G. Turaev, Quantum Invariants of Knots and 3-Manifolds (de Gruyter, New York 1994).

[2] B. Bakalov and A.A. Kirillov, Lectures on Tensor Categories and Modular Functors (AMS, Providence 2000).

[3] G. Moore and N. Seiberg, Commun. Math. Phys. 123, 177 (1989).

[4] J. Cardy, Nucl. Phys. B270, 186 (1986).

[5] E. Verlinde, Nucl. Phys. B300, 360 (1988).

[6] J. Fuchs, I. Runkel, C. Schweigert, Nucl.Phys. B646, 353 (2002). 
[7] J. Fuchs, I. Runkel, C. Schweigert, hep-th/0110158.

[8] J. Fuchs, C. Schweigert, Fields Inst. Comm. 39, 25-70 (2003).

[9] A.N. Schellekens, S. Yankielowicz, Phys.Lett.B227, 387 (1989).

[10] A.N. Schellekens, S. Yankielowicz, Nucl.Phys.B327, 673 (1989).

[11] A.N. Schellekens, S. Yankielowicz, Int.J.Mod.Phys.A5, 2903 (1990).

[12] K.A. Intriligator, Nucl.Phys.B332, 541 (1990).

[13] B. Gato-Rivera, A.N. Schellekens, Commun.Math.Phys.145, 85 (1992).

[14] M. Kreuzer, A.N. Schellekens, Nucl.Phys.B411, 97 (1994).

[15] F. Gliozzi, J. Scherk and D. I. Olive, Phys.Lett. B65, 282 (1976).

[16] F. Gliozzi, J. Scherk and D. I. Olive, Nucl.Phys. B122, 253 (1977).

[17] J. Fuchs, C. Schweigert, J. Walcher, Nucl.Phys. B588, 110 (2000).

[18] J. Fuchs, A.N. Schellekens and C. Schweigert, Nucl.Phys. B473, 323 (1996).

[19] P. Bantay, JHEP03(2003)025.

[20] M.B.Halpern, Ann. of Phys. 194, 247 (1989).

[21] J. Fuchs, I. Runkel, C. Schweigert, hep-th/0403157.

[22] T. Gannon, math.qa/0103044

[23] P. Bantay, Int.J.Mod.Phys. A13, 199 (1998).

[24] J. de Boere and J. Goeree, Commun. Math. Phys. 139, 267 (1991).

[25] A. Coste and T. Gannon, Phys. Lett. B323, 316 (1994).

[26] P. Bantay, Commun. Math. Phys. 233, 423 (2003).

[27] G.M. Ziegler, Lectures on polytopes (GTM 152, Springer-Verlag, 1994).

[28] The GAP Group, GAP - Groups, Algorithms, and Programming, Version 4.4; 2004. (http://www.gap-system.org).

[29] K. Fukuda, cdd+-077 (http://www.cs.mcgill.ca/〜 fukuda/soft/cdd_home/cdd.html). 